\title{
Cyst aspiration with gonadotropin suppression in ovarian remnant syndrome with ureteral obstruction: A case report
}

\author{
David J. DeWitt ${ }^{1}$, Kathleen Eyster ${ }^{2}$, Keith A. Hansen ${ }^{3 *}$ \\ ${ }^{1}$ PGY-1 Obstetrics and Gynecology, Grand Rapids Medical Partners, Grand Rapids, USA \\ ${ }^{2}$ Division of Basic Biomedical Sciences, Department of Obstetrics and Gynecology, Sanford School of Medicine, The University of \\ South Dakota, Vermillion, USA \\ ${ }^{3}$ Sanford Women's Health, Department of Obstetrics and Gynecology, Sanford School of Medicine, The University of South Dakota, \\ Sioux Falls, USA \\ Email: *kahansen@usd.edu
}

Received 3 August 2012; revised 3 September 2012; accepted 12 September 2012

\begin{abstract}
Background: Ovarian remnant syndrome (ORS) is characterized by functional ovarian tissue following bilateral salpingo-oophorectomy. ORS presents with pelvic pain or mass, but may also present with urinary tract symptoms resulting from enlarging residual tissue. Case: A 37-year-old woman presented with pelvic pain radiating to her flank due to ureteral obstruction from an enlarging ovarian cyst in ORS. Transvaginal ultrasound guided, ovarian cyst aspiration accompanied by leuprolide acetate gonadotropin suppression resulted in acute and chronic relief of ureteral obstruction. Conclusion: This case demonstrated successful treatment of ureteral obstruction caused by an ovarian cyst in a patient with ORS via cyst aspiration and leuprolide acetate gonadotropin suppression. This treatment is a viable alternative for management of ORS, but sacrifices pathologic diagnosis when compared to traditional surgical resection.
\end{abstract}

Keywords: Ovarian Remnant Syndrome; Ureteral Obstruction; Leuprolide Acetate

\section{INTRODUCTION}

Ovarian remnant syndrome (ORS) is defined as functioning ovarian tissue left inadvertently at the time of bilateral salpingo-oophorectomy (BSO) and has been associated with pelvic pain, a mass, or ureteral obstruction [1]. ORS results when remnants of ovarian cortex, remaining on the peritoneal surface of the pelvis, become functional following revascularization [2,3]. Identified risk factors for ORS include a history of endometriosis, pelvic inflammatory disease, multiple previous opera-

${ }^{*}$ Corresponding author. tions and pelvic adhesive disease [3]. It has been reported that patients with ORS had 1 to 9 previous operations, with a mean of 4.3 surgeries [4].

Follicle Stimulating Hormone (FSH) levels may be helpful in the diagnosis of ORS with premenopausal levels in a woman following BSO suggestive of the presence of functional remnants of ovarian tissue [1]. On the other hand, elevated FSH levels do not rule out ovarian remnant syndrome. In this case clomiphene citrate or a $\mathrm{GnRH}$ agonist may assist in making the diagnosis by stimulating residual ovarian fragments and make them easier to identify on imaging or at surgery [5]. The initial injection of a GnRH agonist causes a transitory release of gonadotropins prior to suppression. The definitive diagnosis of ORS is made with the pathological confirmation of residual ovarian tissue at a subsequent surgery following BSO.

Traditional treatment of ORS entails surgical removal by laparotomy, or more recently laparoscopy, with wide excision of the mass, with a recurrence rate of about $10 \%$ $[1,3]$. Complications of surgical removal of ORS include enterotomy/colotomy (5\%), cystotomy (2\%), ureteral injury (1\%), blood loss requiring transfusions (12\%), thromboembolic events including pulmonary emboli or deep venous thrombosis (2\%), adynamic ileus or partial small-bowel obstruction (8\%), wound infection (4\%), abdominal abscess (1\%), pneumonia (1\%), sepsis (2\%), and ureteral obstruction or stricture $(2 \%)$ [3,5]. The purpose of this case study is to describe an alternative, minimally invasive method for treating ORS, with transvaginal ultrasound guided cyst aspiration accompanied by gonadotropin suppression with leuprolide acetate.

\section{CASE REPORT}

A 37-year-old woman presented with left lower quadrant 
pain, described as intermittent, sharp and radiating through to her flank. A computer tomography scan demonstrated left hydronephrosis with a simple, cystic mass on the left pelvic sidewall, close to the area of ureteral obstruction. Cystoscopy with retrograde pyelograms was performed (Figure 1), followed by placement of a left ureteral stent. Thirty months prior to this presentation an abdominal hysterectomy and right salpingo-oophorectomy was performed for menorrhagia and dysmenorrhea secondary to adenomyosis. Subsequent to this surgery the patient developed intermittent left lower quadrant pain due to ultrasound documented, ovarian cysts. Five months prior to the current presentation, she had a laparoscopic left salpingo-ophorectomy. During the laparoscopic surgery extensive adhesions were revealed and during lysis of adhesions the bowel was inadvertently injured and repaired. Pathology revealed a simple, functional ovarian cyst. Five months after the left salpingooophorectomy the patient presented with left lower quadrant pain radiating to her flank, and was discovered to have left hydronephrosis. Transvaginal ultrasound demonstrated a 3 centimeter, simple appearing left adnexal cyst on the left pelvic sidewall which was adjacent to and appeared to be compressing the left ureter (the left ureter was able to be visualized due to the presence of the ureteral stent). Since the pathology from pre-

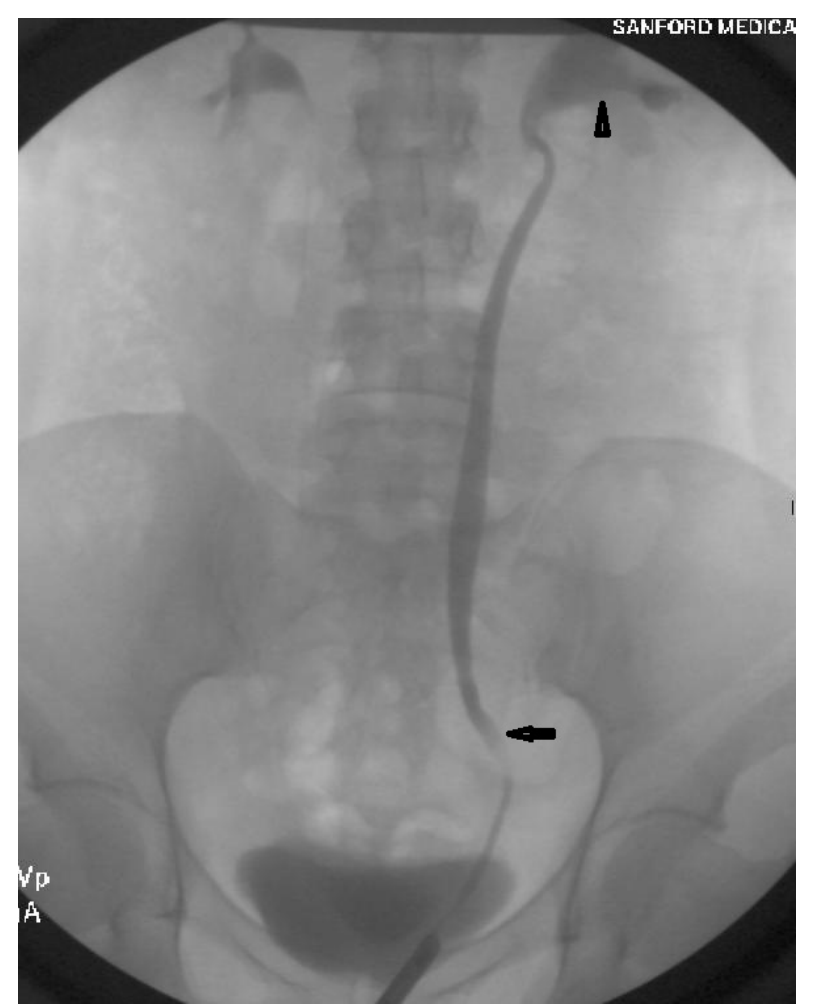

Figure 1. Left hydroureter and left hydronephrosis on retrograde pyelogram at 5 months following removal of her left ovary. Arrow points to left hydroureter just above area of obstruction. Arrowhead identifies hydronephrotic left kidney. vious surgeries had demonstrated benign ovarian cysts and ovaries, and the current cyst was simple in appearance on ultrasound, it was felt that transvaginal, ultrasound guided cyst aspiration was a viable option for acute relief of ureteral obstruction, rather than the traditional surgical resection. The patient was counseled regarding transvaginal ultrasound guided cyst aspiration versus laparoscopy, possible laparotomy with removal of the ovarian remnants. Following discussion of benefits and risks of both procedures the patient elected to proceed with cyst aspiration. In the operating room, she was placed under general anesthesia. A transvaginal ultrasound was performed followed by ultrasound guided cyst aspiration using a 17-gauge single-lumen catheter which obtained clear fluid. The cyst wall collapsed and concomitantly the left ureter sonograhically dilated to normal size. Leuprolide acetate was immediately started following the cyst aspiration to prevent cyst recurrence. The ureteral stent was successfully removed two weeks post operative. The patient has not had a recurrence of pain or ureteral obstruction from the ovarian remnant syndrome while treated with leuprolide acetate at 6 months follow up. Currently, the patient is deciding whether to undergo surgical resection or continue leuprolide acetate.

\section{DISCUSSION}

This case illustrates a minimally invasive option in the armamentarium for management of ORS. In this case, ureteral obstruction presumed secondary to an ovarian cyst forming in an ovarian remnant was treated with transvaginal ultrasound-guided, cyst aspiration with concomitant leuprolide acetate gonadotropin suppression. Ureteral patency was reestablished with relief of her pain and no recurrence of ORS for six months. In this case the motivation to perform cyst aspiration and leuprolide acetate was to reduce the risks associated with surgical management in a patient with severe pelvic adhesive disease. One risk of using cyst aspiration as opposed to surgical resection is inadvertently aspirating a malignant cyst and, potentially spreading the disease as well as delaying the diagnosis. In this case transvaginal ultrasound guided cyst aspiration was considered a reasonable option considering the patient's benign pathology in two previous surgeries and the appearance of a unilocular, simple cyst on ultrasound. Alternative, noninvasive or minimally invasive methods for treating ORS have been previously described. A previous case demonstrated successful treatment with leuprolide acetate of recurrent ORS resistant to past surgical interventions which presented with ureteral obstruction [5]. Previous studies have used oral contraceptive pills, danazol, gonadotropin-releasing hormone agonists and radiotherapy in the 
treatment of resistant ORS [2,5].

This case report demonstrated the complication of ovarian remnant syndrome following traditional removal of the ovaries by blunt dissection followed by transection of the infundibulopelvic ligament. Magtibay et al. described removal of the ovaries with surrounding peritoneum/visceral serosa to potentially reduce the risk of ORS. This en masse removal of the ovaries is accomplished by entering the retroperitoneal space lateral to the ovaries and ligating the ovarian vessels high, cephalad to the ovaries. This en masse technique for removal of the ovaries reduces the risk of leaving remnants of ovarian tissue behind and reduces the risk of developing ORS [3].

In ORS the leuprolide acetate should be continued until after menopause, when the ovarian remnants will be severely diminished or devoid of follicles and have decreased risk of forming a functional ovarian cyst. The hypoestrogenic affects of leuprolide acetate are similar to the risks following surgical menopause. The risks and long term costs of chronic leuprolide acetate therapy should be weighed against the risks and cost of surgery, and discussed with the patient prior to initiation of this therapy. In using alternative treatment options compared to surgical resection, the provider relinquishes the knowledge of a definitive tissue diagnosis. The risk of the missing tissue diagnosis is the possibility of missing cancer, so one must be careful in selecting the patient for a transvaginal ultrasound guided cyst aspiration

\section{CONCLUSION}

This case illustrates a minimally invasive technique for potential use in patients with ORS. Cyst aspiration should be undertaken only when the practitioner has a high suspicion of a benign lesion, in this case a history of benign pathology and simple appearing cyst on ultrasound. In addition, in high risk patients a retroperitoneal approach to salpingo-oophorectomy rather than the traditional transection of the infundibulopelvic ligament may reduce the occurrence of ORS. Further studies will need to be completed to more definitively establish this treatment as a viable alternative in terms of safety and recurrence of ORS.

\section{REFERENCES}

[1] Katz, V.L., Ed. (2007) Ovarian remnant syndrome. Katz: Comprehensive Gynecology, 5th Edition, Chapter 18, Mosby, Philadelphia.

[2] Bryce G.M. and Malone, P. (1989) The ovarian remnant syndrome presenting with acute urinary retention. Postgraduate Medical Journal, 65, 797-798. doi:10.1136/pgmj.65.768.797

[3] Magtibay, P.M., Nyholm, J.L., Hernandez, J.L. and Podratz, K.C. (2005) Ovarian remnant syndrome. American Journal of Obstetrics \& Gynecology, 193, 2062-2066. doi:10.1016/j.ajog.2005.07.067

[4] Siddall-Allum, J. (1994) The ovarian remnant syndrome. Journal of the Royal Society of Medicine, 87, 375-376.

[5] Koch, M.O., Coussens, D. and Burnett, L. (1994) The ovarian remnant syndrome and ureteral obstruction: Medical management. Journal of Urology, 152, 158-160. 